\title{
Ocorrência de adenomas pituitários correlacionados a idade e sexo em centros de referências para tratamento neurocirúrgico de patologia selar
}

\author{
Marcelo Lemos Vieira da Cunha1, Matheus Lemos Vieira da Cunha², Denildo César Amaral \\ Veríssimo ${ }^{3}$, Roberta Rehder ${ }^{3}$, Luis Alencar Biurrum Borba ${ }^{4}$, Cesar Luiz Boguszewski ${ }^{5}$
}

Departamento de Neurocirurgia do Hospital Universitário Evangélico de Curitiba, PR, Brasil.

\section{RESUMO}

Objetivo: Avaliar a incidência dos adenomas pituitários (AP) em centros de referência no tratamento da patologia selar, em uma área geográfica bem delimitada, analisando-os de acordo com o sexo e a idade dos pacientes no momento do ato neurocirúrgico. Métodos: Realizado estudo retrospectivo dos pacientes submetidos a tratamento neurocirúrgico via transesfenoidal realizado pelo neurocirurgião sênior (LABB), com imunoistoquímica compatível com adenoma hipofisário. Resultados: Entre os 231 casos selecionados, os adenomas pituitários não funcionantes foram os mais comuns. Em relação às lesões ditas funcionantes, houve predomínio dos produtores do hormônio do crescimento (GH). $A$ superioridade dos não funcionantes em números absolutos e do GH no subgrupo das lesões produtoras é válida tanto na análise geral dos dados quanto na estratificação da população estudada por sexo. Os pacientes foram agrupados, conforme a idade, em décadas de vida, com predomínio de doentes tratados cirurgicamente entre 30 e 40 anos. A relação masculino:feminino foi de 1:1,69. Conclusão: $A$ escassez de dados não permite uma análise global mais fidedigna da distribuição dos adenomas pituitários com referência ao subgrupo, idade e sexo. Nossos dados são destoantes dos de outras publicações em alguns dos aspectos analisados, o que pode conotar diferenças epidemiológicas regionais dos AP.

\section{PALAVRAS-CHAVE}

Hipófise, epidemiologia, procedimentos neurocirúrgicos.

\section{ABSTRACT}

Occurrence of pituitary adenomas correlated with age and gender in reference centers for neurosurgical sellar pathology

Objective: To evaluate the incidence of pituitary adenomas $(P A)$ in reference centers for the treatment of sellar disease in a well-defined geographical area, analyzing them according to sex and age of patients at the time of the neurosurgery. Methods: A retrospective study of patients undergoing transsphenoidal neurosurgical made by senior neurosurgeon (LABB), with immunohistochemistry consistent with pituitary adenoma. Results: Of the 231 selected cases, nonfunctioning pituitary adenomas were the most common. In relation to the functioning lesions happens a predominance of the producers of growth hormone (GH). The superiority of nonfunctioning in absolute numbers and in the subgroup of GH-producing lesions is valid both in the overall analysis of the data, and the stratification of the study population by sex. Patients were grouped according to age in decades of life, with a predominance of patients treated surgically between 30 and 40 years. The male: female ratio was 1:1,69. Conclusion: The lack of data does not allow a more accurate overall assessment of the pituitary adenomas distribution with respect to the subgroup, age and sex. Our data are divergent from other publications in some of the aspects analyzed, which may connote regional epidemiological differences of AP.

\section{KEYWORDS}

Pituitary gland, epidemiology, neurosurgical procedures.

Neurocirurgião do Hospital Regional do Oeste, Chapecó, SC, Brasil.

2 Acadêmico do curso de Medicina da Universidade do Vale do Itajaí (Univali), Vale do Itajaí, SC, Brasil.

3 Médico(a)-residente em Neurocirurgia do Hospital Universitário Evangélico de Curitiba, PR, Brasil.

Chefe do Departamento de Neurocirurgia do Hospital Universitário Evangélico de Curitiba, PR, Brasil.

5 Doutor em Endocrinologia, professor adjunto do Departamento de Clínica Médica e chefe do Serviço de Endocrinologia e Metabologia do Hospital de Clínicas da Universidade Federal do Paraná (SEMPR), Curitiba, PR, Brasil. 


\section{Introdução}

Os tumores pituitários se originam primariamente na glândula pituitária anterior (adenoipófise). Tumores da neuroipófise são raros. Os adenomas pituitários (AP) representam 15\%-19\% dos tumores encefálicos primários tratados cirurgicamente. ${ }^{1-3}$ Segundo estatísticas norte-americanas, a incidência anual estimada é de 8,2 a 14,7 casos por 100.000 indivíduos. Esses números fazem dos AP o terceiro mais comum tumor intracraniano primário, atrás apenas dos gliomas e meningiomas. ${ }^{4} \mathrm{E}$ é possível que os novos casos de AP cresçam ainda mais. Um estudo realizado no estado norte-americano da Califórnia demonstrou maior incidência dos adenomas hipofisários no período de 2001 a 2005. Possivelmente, esse aumento se deve à maior quantidade de crianças expostas à radioterapia. O período médio de desenvolvimento dos AP gira em torno de 30 anos após a radiação. ${ }^{5}$ Se considerarmos estudos de autópsias, $20 \%$ a $27 \%$ da população possui AP subclínicos, os chamados "incidentalomas". ${ }^{6}$ Adenomas ocultos clinicamente podem ser demonstrados em até $10 \%$ das ressonâncias magnéticas realizadas rotineiramente. ${ }^{7}$

Como sugerem os dados supracitados, é cada vez maior a possibilidade de o neurocirurgião se defrontar em sua prática diária com pacientes portadores de AP. Cabe, então, um melhor conhecimento acerca dessa moléstia pela nossa classe.

\section{Materiais e métodos}

Uma análise retrospectiva de prontuários dos pacientes submetidos a tratamento cirúrgico via transnasal transesfenoidal pelo neurocirurgião sênior (LABB) para tratamento de lesão expansiva selar foi realizada a partir de janeiro de 2005 até outubro de 2011. Os dados foram compilados no setor de arquivo médico de três instituições hospitalares de Curitiba, Paraná, onde ele realiza os procedimentos neurocirúrgicos. Dos 288 pacientes selecionados, foram considerados apenas os que possuíam perfil imunoistoquímico condizente com adenoma pituitário, totalizando 231 indivíduos.

Os dados encontrados foram analisados observando-se as variáveis: idade, sexo e subtipo histológico tumoral. Os tumores também foram delineados, conforme status hormonal do paciente, em funcionantes e não funcionantes. Pacientes com perfil imunoistoquímico compatível com mais de um hormônio produtor foram agrupados sob a nômina pluri-hormonais.

As referências literárias foram obtidas por meio de pesquisa eletrônica no PubMed (www.pubmed.org), mediante procura dos termos: "pituitary epidemiology", "pituitary" e "pituitary adenoma”, sem restrições. Os dados considerados pertinentes serão discutidos e comparados com os dados levantados.

\section{Resultados}

Durante o período de janeiro de 2005 a outubro de 2011, 231 pacientes submetidos a tratamento neurocirúrgico com o diagnóstico imunoistoquímico de adenoma pituitário foram selecionados. Desses, 86 $(37,23 \%)$ eram do sexo masculino e $145(62,78 \%)$, do sexo feminino, o que nos confere uma relação feminino:masculino de 1,69:1. A idade média na data do procedimento cirúrgico foi de 43,78 anos. A idade mínima registrada foi a de um paciente do sexo masculino portador de lesão secretora de GH: 10 anos. A idade máxima foi a de um paciente do sexo feminino com tumor não secretor: 81 anos. $O$ maior número de cirurgias ocorreu quando os pacientes se encontravam na quarta década de vida (60 de 231, 25,97\%) (Tabela 1).

Da população total considerada no estudo, houve predomínio dos tumores funcionantes (158 de 231, $68,40 \%$ ) sobre os não funcionantes ( 73 de $231,31,60 \%$ ). Considerando-se somente os funcionantes, houve predomínio dos produtores de GH (64 de 158, 40,50\%) (Figura 1).

\begin{tabular}{lcc}
\hline & $\begin{array}{c}\text { Tabela 1 - Distribuição dos adenomas } \\
\text { pituitários por idade (231 casos) }\end{array}$ \\
\hline Idade (anos) & Número & Percentagem \\
\hline $0-20$ & 7 & 3,03 \\
$21-30$ & 50 & 21,64 \\
$31-40$ & 60 & 25,97 \\
$41-50$ & 46 & 19,92 \\
$51-60$ & 46 & 19,92 \\
$>60$ & 22 & 9,52 \\
\hline
\end{tabular}

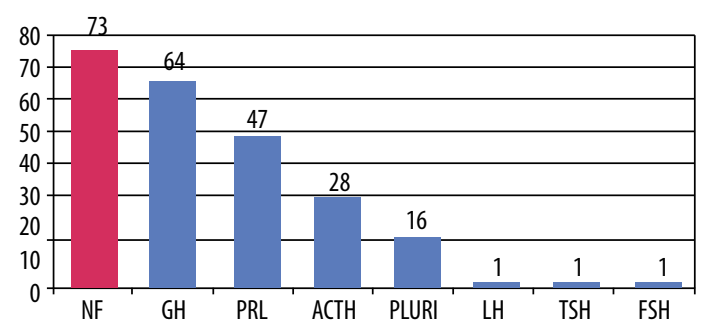

Figura 1 - Distribuição dos subtipos de adenomas hipofisários conforme laudo imuno-histoquímico. 
Os pluri-hormonais englobam 16 pacientes, dos quais: oito são produtores concomitantemente de hormônio do crescimento $(\mathrm{GH})$ e prolactina (PRL); três são coprodutores de GH e hormônio adrenocorticotrófico (ACTH); dois são produtores de hormônio luteinizante (LH) e folículo-estimulante (FSH); um é produtor de GH e hormônio estimulante da tireoide (TSH); um produz LH e TSH; e, por fim, um é produtor de PRL e FSH.

Com relação à população masculina estudada, foram registrados 86 casos. A idade média foi de 44,88 anos, com amplitude de 10 a 79 anos. Os tumores funcionantes foram maioria, representando $61,63 \%$ dos casos (53 de 86). Nesse universo, os produtores de GH correspondem a 49,05\% (26 de 53); 12 correspondem a leões produtoras de ACTH (22,5\%); oito possuíam imunoistoquímica compatível com prolactinoma (15,10\%); um caso registrado de $\mathrm{LH}(1,89 \%)$ e em seis casos houve detecção de mais de um hormônio, caracterizando a categoria pluri-hormonal $(11,32 \%)$. Os tumores não funcionantes perfizeram $38,37 \%$ (33 de 86 ).

Na população feminina encontramos a maioria dos casos: 145 . A idade média atingiu 42,25 anos, com variação de 17 a 81 anos. Houve, também, predomínio dos tumores funcionantes em relação aos não funcionantes. No entanto, o maior volume registrado em mulheres foi de prolactinomas (Tabela 2). Os não funcionantes atingiram 45 casos $(31,03 \%)$, enquanto os funcionantes corresponderam a 100 casos $(68,97 \%)$.

\begin{tabular}{lcc}
\hline & $\begin{array}{c}\text { Tabela 2 } \\
\text { adenomas pituitários no sexo }\end{array}$ \\
\hline Subgrupo & No de casos $^{\circ}$ & Percentagem \\
\hline NF & 45 & 31,03 \\
PRL & 38 & 26,21 \\
GH & 35 & 24,83 \\
ACTH & 15 & 10,34 \\
PLURI & 9 & 6,21 \\
TSH & 1 & 0,69 \\
FSH & 1 & 0,69 \\
\hline
\end{tabular}

NF: não funcionante; PRL: prolactina; GH: hormônio do crescimento; ACTH: hormônio adrenocorticotrófico; PLURI: pluri-hormonal; TSH: hormônio estimulante da tireoide; FSH: hormônio folículo estimulante.

\section{Discussão}

Os dados epidemiológicos formam a base do sistema de saúde. Esses dados projetam ações para o controle de determinada moléstia, formando os pilares para uma otimização das políticas de saúde, sobretudo em países com recursos escassos. Os dados da incidência na população dos adenomas pituitários em relação ao sexo e idade são ínfimos, mesmo em países ditos desenvolvidos. Nestes, dados oriundos da análise de imagens na RM encéfalo e baseados em autópsias para determinar a incidência dos adenomas pituitários são discordantes. ${ }^{8}$ Os adenomas pituitários ocorrem em todas as idades. No entanto, sua maior incidência está entre a terceira e a sexta década - fato corroborado em nossa análise -, com os tumores funcionantes prevalecendo em pacientes mais jovens. ${ }^{1}$

A incidência dos tumores não funcionantes aumenta conforme aumenta a idade. ${ }^{7}$ De acordo com Goel et al., ${ }^{9}$ $70 \%$ dos adenomas pituitários são endocrinologicamente ativos, isto é, funcionantes.

Este manuscrito oferece uma análise imunoistoquímica da incidência local de adenomas hipofisários. Mesmo que geralmente benignos, os AP estão associados à substancial morbidade. ${ }^{10} \mathrm{O}$ conhecimento local permite o entendimento de possíveis diferenças epidemiológicas e mudanças nas ações de saúde local e global, justificando diferentes ações em diferentes espaços geográficos para uma mesma patologia.

Por exemplo, em contraste ao relato de Shamim et al., ${ }^{1}$ as mulheres obtiveram ampla superioridade em relação aos homens na nossa série $(62,78 \%$ versus $37,23 \%)$. No entanto, nossa série avaliza os números encontrados em outras publicações que apontam os adenomas pituitários como uma patologia com predominância no sexo feminino. ${ }^{6,8,11,12}$ Uma das possíveis explicações para essa preponderância masculina na série de Shamim et al. reside no fato de essa série basear-se em um país do sul asiático, onde os homens costumam receber maiores cuidados médicos que as mulheres, conforme exposto pelo próprio autor.

Conforme demais séries analisadas, ${ }^{11-13}$ a idade média dos pacientes femininos com adenomas hipofisários (42,25 anos) é menor do que a dos pacientes masculinos (44,88 anos). Esse fato deve ser acompanhado com atenção, pois Raappana et al. ${ }^{8}$ verificaram um segundo pico de incidência em mulheres acima dos 70 anos em séries recentes, carreando um maior índice de suspeição clínica e cuidados no ato cirúrgico quando necessário. Em nossa série, destoando da análise de Raappana et al. ${ }^{8}$ pacientes acima dos 60 anos atingiram uma porcentagem condizente com demais séries pesquisadas: 9,52\% (22 de 231). . $^{8,11,14}$

Relativamente aos adenomas funcionantes, a maior parte das séries aponta os prolactinomas como subgrupo dominante. ${ }^{8,11}$ No entanto, sabe-se que grande parte desse subgrupo necessita apenas de tratamento clínico para controle da doença, contrastando com outros subtipos de adenomas funcionantes que possuem no tratamento cirúrgico sua primeira opção de tratamento.

Em nossa série, foram considerados apenas pacientes submetidos a tratamento neurocirúrgico, conferindo uma característica peculiar à população estudada. O subgrupo dos produtores de $\mathrm{GH}$, conforme aponta a 
tabela 2, foi preponderante perante os demais subtipos de lesões funcionantes. Se comparado com outras séries encontradas na literatura, esse dado não é uniforme, podendo variar conforme população e idade estudadas.

No estudo de Dyer et al., ${ }^{6}$ por exemplo, os autores estudaram pacientes menores que 16 anos, submetidos à cirurgia transesfenoidal para adenomas pituitários, em país europeu, verificando uma ampla maioria de crianças com tumores funcionantes do subgrupo ACTH.

Já na série de Mindermann e Wilson, ${ }^{11}$ o subtipo predominante foi de prolactinoma na população estudada tratada neurocirurgicamente, seguido dos não funcionantes. Nesse estudo houve predomínio da população entre a segunda e a quinta década de vida.

\section{Conclusão}

Há poucos estudos epidemiológicos da incidência de adenomas pituitários baseados na população. Assim, há limitado conhecimento sobre a real incidência global e dos subgrupos de adenomas hipofisários. Dados recentes sobre a incidência das lesões tumorais hipofisárias são necessários para complementar os dados de prevalência já existentes, a fim de entender a situação presente e lançar perspectivas para o futuro das necessidades inerentes ao tratamento dos adenomas pituitários pelo sistema de saúde. A incidência dos AP varia conforme a localidade estudada. Esta publicação fornece um estudo atualizado sobre a incidência dos adenomas pituitários em uma comunidade neurocirúrgica numa área geográfica bem definida. $\mathrm{O}$ conhecimento regionalizado permite melhor assistência à população local, com melhores resultados: objetivo comum a neurocirurgiões e pacientes.

\section{Referências}

1. Shamim MS, Bari ME, Khursheed F, Jooma R, Enam SA Pituitary adenomas: presentations and outcomes in a South Asian country. Can J Neurol Sci. 2008;35(2):198-203.

2. Fontana E, Gaillard R. Epidemiology of pituitary adenoma: results of the first Swiss study. Rev Med Suisse. 2009;5(223):2172-4.

3. Annegers JF, Coulam CB, Abboud CF, Laws ER Jr, Kurland LT. Pituitary adenoma in Olmsted County, Minnesota, 1935-1977. A report of an increasing incidence of diagnosis in women of childbearing age. Mayo Clin Proc. 1978;53(10):641-3.

4. Cardoso ER, Peterson EW. Pituitary apoplexy: a review. Neurosurgery. 1984;14(3):363-73.

5. Nasseri K, Mills JR. Epidemiology of primary brain tumors in the Middle Eastern population in California, USA 2001 2005. Cancer Detect Prev. 2009;32(5-6):363-71.

6. Dyer EH, Civit T, Visot A, Delalande O, Derome P. Transsphenoidal surgery for pituitary adenomas in children. Neurosurgery. 1994;34(2):207-12.

7. Chong BW, Kucharczyk W, Singer W, George S. Pituitary gland MR: a comparative study of healthy volunteers and patients with microadenomas. AJNR Am J Neuroradiol. 1994;15(4):675-9.

8. Raappana A, Koivukangas J, Ebeling T, Pirilä T. Incidence of pituitary adenomas in Northern Finland in 1992-2007. J Clin Endocrinol Metab. 2010;95(9):4268-75.

9. Goel A, Nadkarni T, Muzumdar D, Desai K, Phalke U, Sharma P. Giant pituitary tumors: a study based on surgical treatment of 118 cases. Surg Neurol. 2004;61(5):436-45.

10. Schoemaker MJ, Swerdlow AJ. Risk factors for pituitary tumors: a case-control study. Cancer Epidemiol Biomarkers Prev. 2009;18(5):1492-500.

11. Mindermann T, Wilson CB. Age-related and gender-related occurrence of pituitary adenomas. Clin Endocrinol (Oxf). 1994;41(3):359-64.

12. Yamada S, Kovacs K, Horvath E, Aiba T. Morphological study of clinically nonsecreting pituitary adenomas in patients under 40 years of age. J Neurosurg. 1991;75(6):902-5.

13. Partington MD, Davis DH, Laws ER Jr, Scheithauer BW. Pituitary adenomas in childhood and adolescence. Results of transsphenoidal surgery. J Neurosurg. 1994;80(2):209-16.

14. Daly AF, Rixhon M, Adam C, Dempegioti A, Tichomirowa MA, Beckers A. High prevalence of pituitary adenomas: a cross-sectional study in the province of Liege, Belgium. J Clin Endocrinol Metab. 2006;91(12):4769-75.

Endereço para correspondência

Marcelo Lemos Vieira da Cunha

Rua Rui Barbosa, 93 E, ap. 801, Centro

89801-040 - Chapecó, SC, Brasil

E-mail: marcelolvc@yahoo.com.br 